Cortical Bone Viability

\title{
Improved Autologous Cortical Bone Harvest and Viability with 2Flute Otologic
}

\section{Burs}

Adam A. Roth, BS ${ }^{1}$, Pei-Ciao Tang, $\mathrm{PhD}^{1}$, Michael J. Ye, BA², Khalid S. Mohammad, MD, $\mathrm{PhD}^{2}$, Rick F. Nelson, $\mathrm{MD}, \mathrm{PhD}^{1}$

Author Affiliation:

${ }^{1}$ Department of Otolaryngology—Head and Neck Surgery

${ }^{2}$ Department of Medicine

Indiana University School of Medicine, Indianapolis, IN 46202

Disclosures: $\mathrm{RN}$ is a paid consultant for Stryker Corporation, but received no compensation for this study.

Funding: IU School of Medicine, Department of Otolaryngology Head and Neck Surgery

Award

Running Title: Cortical Bone Viability

$\underline{\text { Key words: }}$ Bone, canal wall reconstruction tympanomastoidectomy, viability, cholesteatoma, autologous, burs, drill, healing, mastoidectomy

Level of Evidence: NA

Word Count: 2685

Corresponding author:

Rick F. Nelson

355 W. $16^{\text {th }}$ Street, Suite 3200

Indianapolis, IN, 46202

Phone: 317-963-7073; Fax: 317-963-7085; ricnelso@iupui.edu

This study has been has been selected for poster presentation for the Triological Society at the 120th Annual Meeting at COSM to be held April 28-29, 2017, in San Diego, CA. 
Objectives: To determine if 2Flute otologic burs improve the size, cellular content and bone healing of autologous cortical bone grafts harvested during canal wall reconstruction tympanomastoidectomy with mastoid obliteration.

Study Design: IRB-approved prospective cohort study.

Methods: Human autologous cortical bone chips were harvested using various burs (4 and 6 mm diameter; multiflute and 2Flute) from patients undergoing canal wall reconstruction tympanomastoidectomy for the treatment of chronic otitis media with cholesteatoma. Bone chip size, cell counts, cellular gene expression and new bone formation were quantified.

Results: Bone chips were significantly larger when harvested with 2Flute bur compared to multiflute burs at both $6 \mathrm{~mm}$ diameter $\left(113 \pm 14 \mu \mathrm{m}^{2}\right.$ vs. $\left.66 \pm 8 \mu \mathrm{m}^{2} ; \mathrm{P}<0.05\right)$ and $4 \mathrm{~mm}$ diameter (70 $\pm 8 \mu \mathrm{m}^{2}$ vs. $50 \pm 3 \mu \mathrm{m}^{2}$; $\left.\mathrm{P}<0.05\right)$. After 2 weeks in culture, cell numbers were significantly higher when harvested with 2Flute bur compared to multiflute burs at both 6 mm diameter (48.7 \pm 3 vs. $31.8 \pm 3$ cells/ $\mu$ g bone; $\mathrm{P}<0.05)$ and 4 mm diameter $(27.6 \pm 1.2$ vs. $8.8 \pm 1.2$ cells $/ \mu g$ bone; $\mathrm{P}<0.05)$. Bone derived cells express osteoblast markers (alkaline phosphatase, osteocalcin). Cultured cells are able to form new bone in culture and bone formation is facilitated by the presence of bone chips.

Conclusions: Use of 2Flute otologic burs for human autologous cortical bone harvest results in more viable bone fragments with larger bone chips and more osteoblasts. Future studies are needed to determine if this leads to improved bone healing. 


\section{Introduction}

Canal wall reconstruction tympanomastoidectomy with mastoid obliteration (CWR) is used for the treatment of cholesteatoma in children and adults. The advantages of CWR include decreased recurrence of cholesteatoma compared to canal wall up (CWU) tympanomastoidectomy, increased intraoperative visualization of cholesteatoma similar to canal wall down (CWD) tympanomastoidectomy, while obviating the need for lifetime mastoid cavity cleanings or water activity restrictions ${ }^{1}$.

The CWR procedure involves harvesting autologous bone pâté from the mastoid or occipital cortex, which is used to obliterate the mastoidectomy defect after the ear canal reconstruction $^{2}$. To increase visualization during cholesteatoma removal, strategic cuts are made in the posterior ear canal allowing it to be removed. After cholesteatoma removal and tympanoplasty, the canal wall is replaced and the mastoid is obliterated with the autologous bone pâté. Obliteration of the mastoid physically supports the reconstructed posterior ear canal and reduces the nitrogen absorbing mucosa of the mastoid epithelium, which has been implicated in the development of cholesteatoma².

Early in the description of the CWR procedure, postoperative mastoid infection was as high as $14.3 \%^{2}$. Subsequently, autologous bone pâté was only harvested from the squamous portion of the temporal bone or the occipital bone without contamination with mastoid air cells and institution of aggressive postoperative antibiotic treatment led to a decrease in the infection rate to $4.5 \%{ }^{2}$.

To date, there is little known about the composition and viability of the bone pâté used to obliterate the mastoid during CWR. Autologous bone pâté is harvested with cutting burs and improving the viability of the bone pâté may also facilitate bone healing and reduce 
Cortical Bone Viability

postoperative infections. Here we investigated how otologic bur size and design impacts the viability of human cortical bone pâté. 


\section{$\underline{\text { Methods }}$}

Our study was IRB-expedited through standard review (IRB Protocol \#: 1502682884). Written consent was obtained for tissue analysis from all patients or their guardians. Our laboratory IBC protocol (IBC \#: IN-675) was also approved.

\section{Harvesting Bone Pâté}

Bone pâté was harvested during CWR tympanomastoidectomy procedures from the squamous portion of the temporal bone or the occipital bones of both adults and children. Samples were collected using four otologic burs in a random order of sequence and were collected only at the start of a drilling procedure to ensure only cortical bone pâté would be collected. Drill burs were provided by Stryker Corporation (Kalamazoo, Michigan). The four different burs included the $6 \mathrm{~mm}$ 2Flute, $4 \mathrm{~mm}$ 2Flute, $6 \mathrm{~mm}$ multiflute, and $4 \mathrm{~mm}$ multiflute. Bone pâté was collected with a sterile tongue blade and placed directly into culture medium. Approximately $10 \mathrm{mg}$ of bone pâté from each bur was placed in a unique $50 \mathrm{~mL}$ conical vial containing $30 \mathrm{~mL}$ of serum-free media (SFM) for transport to a cell culture lab and was plated or fixed within 12 hours after collection. The SFM consisted of Advanced DMEM/F12 with 100 $\mathrm{U} / \mathrm{mL}$ penicillin and $100 \mathrm{ug} / \mathrm{mL}$ streptomycin.

\section{Determination of Bone Fragment Size}

Bone pâté samples were fixed in 10\% neutral-buffered formalin for 48 hours and embedded in OCT. 3.5- $\mu \mathrm{m}$ sections were cut and stained with hematoxylin and eosin (H\&E) with orange G and phloxine. Sections were imaged to determine the bone fragment size distribution for each drill bur type. Samples were de-identified and assigned a random number to blind the imaging and imaging analysis process. All sections were viewed on a Leica DM LB compound microscope outfitted with a Q-Imaging Micropublisher Cooled CCD color digital camera. 
Cortical Bone Viability

Images were captured and analyzed using Bioquant software v. 15.1 (Bioquant Image Analysis

Corp, Nashville , TN). The individual bone fragment surface area measurements were collected into a Prism 6 spreadsheet. Statistical analysis was done using GraphPad Prism program version6. Fragment surface area lengths of $>35 \mu \mathrm{m}^{2}$ were calculated as these represented the largest solitary fragments. A two-tailed Student's t-test was used to determine the significance of the results.

\section{In vitro culture of bone pâté}

Bone pâté samples in SFM medium were placed in a cell culture hood where the SFM was vacuum aspirated. $25 \mathrm{~mL}$ of phosphate buffered saline (PBS) were added to each conical vial. The samples were vortexed for 10 seconds after which the vials were kept still for 30 seconds to allow for any undesired hematopoetic tissue to rise. The PBS was then vacuum aspirated. This process was repeated twice until the bone samples appeared ivory white ${ }^{3}$. All aspirations were performed with care to avoid accidental aspiration of bone pâté.

Uncoated $60 \mathrm{~mm}$ cell culture dishes were plated with $5 \mathrm{~mL}$ of complete media, followed by 10 mg of the bone pâté sample collected with a sterilized microspatula from the conical vial. The complete media consisted of Advanced DMEM/F12 with 10\% fetal bovine serum, 2 mM GlutaMAX, $100 \mathrm{U} / \mathrm{mL}$ penicillin, and $100 \mathrm{ug} / \mathrm{mL}$ streptomycin. The bone pâté culture plates were kept in a $5 \% \mathrm{CO}_{2}, 37^{\circ} \mathrm{C}$ incubator for 3-4 weeks. The media was changed once a week during the first two weeks of culture and twice weekly thereafter. Within 4 weeks the culture specimens were analyzed to understand the cell types present. This analysis was conducted through staining, RT-PCR, and further culture on osteogenic media (PromoCell Mesenchymal Stem Cell Osteogenic Differentiation Medium, Catalog Number: C-28013).

\section{In-vitro Testing of Osteoblast Characteristics}


After 14 days in culture, non-adherent bone pâté fragments were removed from the cell culture plates using a P1000 micropipette with a wide-mouthed tip to leave only adherent cells and adherent bone fragments. Twice, $5 \mathrm{~mL}$ of PBS were carefully added and aspirated via the wide-tipped micropipette to ensure the full removal of all unattached bone fragments. $5 \mathrm{~mL}$ of fresh complete media was restored to the plates.

The osteogenic capacity of the sample was evaluated by replacing the complete media with osteogenic media when the cells reached confluency. After 3 weeks in osteogenic media with twice weekly media replacement the cultures were stained for alkaline phosphatase or with alizarin red to determine calcium/phosphate crystal formation.

For alkaline phosphatase staining the cells were washed once with PBS then fixed with 2 $\mathrm{mL}$ of neutral buffered formalin. After 60 seconds the formalin was aspirated and replaced with a washing buffer of $0.05 \%$ Tween in PBS. The washing buffer was aspirated and replaced with a BCIP/NBT (5-bromo-4-chloro-3-indolyl-phosphate/nitro blue tetrazolium) solution. This solution was incubated in the dark at room temperature for 10 minutes. The plate was washed once with washing buffer followed by PBS. The stain was immediately imaged ${ }^{4}$.

For alizarin red staining the cells were fixed using $2 \mathrm{~mL}$ of $10 \%$ neutral buffered formalin per plate. The plates were then washed twice using $2 \mathrm{~mL}$ of PBS. Alizarin Red (4.1 pH) was added to each plate and kept on the plates for 45 minutes. The stained plates were then washed three times with $2 \mathrm{~mL}$ of ddH20 and immediately imaged ${ }^{4}$.

\section{Counting Cultured Viable Osteoblasts}

After 3-4 weeks of culture, before significant logarithmic growth, the wet bone pâté from each plate was removed through multiple PBS washes and deposited in a pre-weighed container. The bone pâté cultured cells were then trypsinized and passed through a cell strainer to filter out 
Cortical Bone Viability

all bone fragments. These cell suspensions were manually counted using a hemocytometer. The pre-weighed container with discarded bone pâté was then vacuum aspirated, set aside, and dried overnight to obtain a dry bone mass that was used to normalize the cell count data. A two-tailed Student's t-test was used to determine the significance of the results. 


\section{RESULTS}

The four burs used in this study vary by head diameter (6 mm vs. $4 \mathrm{~mm}$ ) and with respect to the cutting design (Figure 1). The 2Flute burs have 2 cutting flutes while the multi-fluted burs have 8 flutes (Figure 1). In addition, the angulation of the flutes around the head of the bur is increased in the multi-fluted burs compared to the 2Flute burs (Figure 1). Finally, the $6 \mathrm{~mm}$ burs have a 50\% larger diameter than the $4 \mathrm{~mm}$ burs.

Bone pâté was harvested from patients undergoing CWR using the 4 various burs. Bone pâté fragments varied in size based on bur size and bur shape. Representative images of bone pâté fragments are shown (Figure 2 A-D). The surface areas of individual bone pâté fragments from each bur were quantified. The $6 \mathrm{~mm}$ 2Flute bur generated the larger bone pâté fragments (Figure 2E). Bone pâté fragments were significantly larger when harvested with 2Flute bur compared to multiflute burs at both $6 \mathrm{~mm}$ diameter $\left(112,632 \pm 14,216 \mu \mathrm{m}^{2}, \mathrm{n}=32 \mathrm{vs} .66,637 \pm\right.$ $8,387 \mu \mathrm{m}^{2}, \mathrm{n}=16$; mean $\left.\pm \mathrm{SE} ; \mathrm{P}<0.05\right)$ and 4mm diameter $\left(70,158 \pm 8,131 \mu \mathrm{m}^{2}, \mathrm{n}=35 \mathrm{vs}\right.$. $50,060 \pm 3,198 \mu \mathrm{m}^{2}, \mathrm{n}=21$, mean $\pm \mathrm{SE} ; \mathrm{P}<0.05$, Figure 2E). Interestingly, bone pâté fragments produced by the $4 \mathrm{~mm}$ 2Flute were not significantly different in size compared to the $6 \mathrm{~mm}$ multi-fluted bur despite having a bur diameter that is 50\% smaller (Figure 2E). This finding implicates that the bur shape (i.e. 2Flute) is integral in obtaining large bone pâté fragments.

Next we analyzed the ability of surviving cells to be cultured from the bone pâté under the 4 bur conditions using equal amounts of bone pâté (Figure 3 A-D). Bur size and shape appears to be correlated with the number of viable bone cells in an autograft. Generally, the adherent cells of the $6 \mathrm{~mm} 2$ flute were more numerous and exhibited sheeting before the other burs. Representative images of each bur after three weeks of in-vitro culture can be seen in Figure 3 A-D. After 3 weeks in culture, cell counts were significantly higher when harvested 
Cortical Bone Viability

with 2Flute burs compared to multiflute burs at both $6 \mathrm{~mm}$ diameter (48.7 \pm 3 vs. $31.8 \pm 3$ cells/ $\mu$ g bone; $\mathrm{P}<0.05)$ and $4 \mathrm{~mm}$ diameter $(27.6 \pm 1.2$ vs. $8.8 \pm 1.2$ cells/ $\mu$ g bone; $\mathrm{P}<0.05$;

Figure 3E). The number of cultured cells was not significantly different between the $4 \mathrm{~mm}$ 2Flute bur and the $6 \mathrm{~mm}$ multiflute bur, despite the bur being 50\% smaller in diameter (Figure 3E).

Next, we analyzed the cells for molecular markers of bone derived cells. Alkaline phosphatase, an enzyme highly expressed in osteoblasts, was observed in most of the cells (Figure 4 A-B). This demonstrates that human autograft bone pâté fragments harbor surviving osteoblasts. Cells derived from all four bur conditions exhibited similar expression of alkaline phosphatase.

We next determined if these cultured cells possessed the ability to generate osteoid or new bone in culture. Bone pâté-derived cells were cultured in an osteogenic media in the presence of bone pâté fragments. At day 31, it appears that the cell density is higher around the bone pâté fragments (Figure 4C, d31). This cellular density expands peripherally around the bone fragment at d45 (Figure 4D, d45). Alizarin Red is a stain used to detect new osteoid or bone formation. We observed new osteoid deposits throughout the plate, but most intensely around each bone pâté fragments (Figure 5E, d46) suggesting that bone formation or cell proliferation is accelerated in the presence of bone pâté fragment. In addition, bone pâté-derived cells cultured in the absence of bone pâté fragments also exhibited Alizarin Red osteoid (data not shown).

Finally, we analyzed the gene expression of the bone pâté-derived cells in culture. Alkaline Phosphatase, Collagen 1a, Osteonectin, and Osteocalcin were all expressed in the 
Cortical Bone Viability

cultured cell (Figure 5). These markers are all highly expressed in osteoblasts with osteocalcin being exclusively expressed by osteoblasts.

Human autologous bone harvested with the $6 \mathrm{~mm}$ 2Flute bur is observed on postoperative imaging of the mastoid after a primary canal wall reconstruction tympanomastoidectomy with mastoid obliteration procedure (Figure 6). 


\section{DISCUSSION}

Bone pâté autografts produced from cortical bone with 2Flute otologic burs contain larger bone fragments than multiflute otologic burs. The 2Flute-harvested bone pâté fragments are not only larger but contain more surviving osteoblasts, which facilitate new bone formation. This suggests that bone derived from 2Flute otologic burs is more viable for replacement into the mastoid during CWR than multiflute otologic burs. This improvement in bioactivity has implications for improved bone healing in addition to potentially decreasing the rates of absorption and infection.

Autografts are often the material of choice when performing cranial reconstruction. In cranioplasty procedures bone autografts are less likely to be infected or resorbed when compared to calcium phosphate bone cement and demineralized bone matrix ${ }^{5}$. Particulate bone pâté autografts are a further refinement reported to reduce osseous defects from 24 percent to 9 percent when compared to split calvarial autografts in cranioplasty ${ }^{6}$. Osteocytes are able to remain viable after drilling and may participate in bone reconstruction after autograft placement. Furthermore, the increased surface area to volume ratio of particulate autografts improves diffusion of nutrients and growth factors to accelerate integration of the autograft ${ }^{7}$.

While the smaller particulate autograft is an improvement over the whole bone autograft, our study suggests increasingly small particulate bone fragments have more limited viability. The 2Flute burs produced larger bone fragments than the multiflute burs. This is likely due to the increased clearance between the 2Flute bur's cutting lips. These relatively large fragments improved the viability of osteocytes as demonstrated by normalized in-vitro cell counts. Fresh autografts have a lower rate of infection and better preservation of desired anatomy than grafts with fewer viable cells such as frozen or pre-harvested grafts ${ }^{5}$. The bone harvest was made 
carefully to ensure the bone pâté was composed of only cortical bone as cancellous bone is more cellular and could skew the results of the cell counts.

The initial phase of bone healing is characterized by neovascularization with increased osteoclast activity and resorption. However, within days osteogenesis will also begin. During the osteogenic period particulate autografts participate in both internal mineralization and creeping mineralization by surrounding bone at the edges of the graft ${ }^{8}$. We observed that particulate bone pâté autografts produce viable cells that may participate in osteogenesis. Our cultured bone pâté cells demonstrate characteristics of osteoblasts including alkaline phosphatase activity, the ability to form osteoid matrix, and osteoblast RNA expression especially when induced by mineralization media. These capacities may be induced by in vivo growth factors during post-surgical bone healing.

Our study's findings are limited by the use of an in-vitro model. In-vitro human osteoblast culture is a powerful, well-studied method with more flexibility than an animal model. Although bone pâté cultures can be stimulated to generate new bone with osteogenic media, autograft osteocytes and osteoblasts may behave differently in vivo. Further study of bone pâté autografts in an animal temporal bone model could further elucidate their post-operative behavior. Also, we did not perform a randomized trial of CWR using various burs to determine bone healing and infections rates. 
Cortical Bone Viability

\section{CONCLUSION}

Innovations in surgical drill bur manufacture have expanded the skull base surgeon's tool kit. In this study we have found that the 2Flute otologic drill bur produces more viable bone fragments. Future studies are need to determine if this leads to improved bone healing. This improvement could be useful in autologous bone graft procedures such as canal wall reconstruction ${ }^{2}$, cranioplasty ${ }^{4-6,9}$, and occipitocervical fusion ${ }^{10,11}$ among others. 
Cortical Bone Viability

\section{FIGURE LEGENDS}

Figure 1. Visual representation of burs tested $(\mathrm{mm}=$ millimeters $)$

Figure 2. (A-D) Representative histologic sections of bone pâté fragments produced by each bur (E) Bone pâté fragment surface area distribution for each bur. ( $\mathrm{mm}=$ millimeters, $*=\mathrm{P}<0.05$, NS = non-significant)

Figure 3. (A-D) Representative images of adherent cells after 2 weeks of culture after harvesting with various burs. (B) Cell counts of adherent cells at 2 weeks in culture of bone pâté fragments obtained with various burs. $(\mathrm{mm}=$ millimeters, $\mu \mathrm{g}=$ micrograms, $*=\mathrm{P}<0.05$, NS $=$ non-significant)

Figure 4. (A, B) Alkaline Phosphatase staining of cultured cells from $6 \mathrm{~mm}$ and $4 \mathrm{~mm}$ 2Flute burs. (C, C', D, D’) In-vitro culture bone cell growth from day 31 to day 45 in culture (Arrows). (E, E') Alizarin red positive osteoid at 46 days in culture. Inset (dashed box) $=40 \mathrm{x}$ magnification. $\mathrm{d}=$ day

Figure 5. RT-PCR analysis of osteoblast related genes from adherent cells from bone pâté fragments after 38 days of culture (bp = base pairs of DNA). L27 is a control gene.

Figure6. Representative sagittal CT image of a patient who has undergone CWR with mastoid obliteration with bone pâté harvested with 6 mm 2Flute bur. Posterior ear canal cuts (arrows) and bone pâté $(*)$ are noted. 


\section{REFERENCES}

1. Walker PC, Mowry SE, Hansen MR, Gantz BJ. Long-term results of canal wall reconstruction tympanomastoidectomy. Otol Neurotol 2014; 35:954-960.

2. Gantz BJ, Wilkinson EP, Hansen MR. Canal wall reconstruction tympanomastoidectomy with mastoid obliteration. Laryngoscope 2005; 115:1734-1740.

3. Gartland A, Rumney RM, Dillon JP, Gallagher JA. Isolation and culture of human osteoblasts. Methods Mol Biol 2012; 806:337-355.

4. Moreira-Gonzalez A, Jackson IT, Miyawaki T, Barakat K, DiNick V. Clinical outcome in cranioplasty: critical review in long-term follow-up. J Craniofac Surg 2003; 14:144-153.

5. Plum AW, Tatum SA. A comparison between autograft alone, bone cement, and demineralized bone matrix in cranioplasty. Laryngoscope 2015; 125:1322-1327.

6. Greene AK, Mulliken JB, Proctor MR, Rogers GF. Primary grafting with autologous cranial particulate bone prevents osseous defects following fronto-orbital advancement. Plast Reconstr Surg 2007; 120:1603-1611.

7. Nam JW, Kim MY, Han SJ. Cranial bone regeneration according to different particle sizes and densities of demineralized dentin matrix in the rabbit model. Maxillofacial plastic and reconstructive surgery 2016; 38:27.

8. Pallesen L, Schou S, Aaboe M, Hjorting-Hansen E, Nattestad A, Melsen F. Influence of particle size of autogenous bone grafts on the early stages of bone regeneration: a histologic and stereologic study in rabbit calvarium. The International journal of oral \& maxillofacial implants 2002; 17:498-506.

9. Grant GA, Jolley M, Ellenbogen RG, Roberts TS, Gruss JR, Loeser JD. Failure of autologous bone-assisted cranioplasty following decompressive craniectomy in children and adolescents. J Neurosurg 2004; 100:163-168.

10. Hwang SW, Gressot LV, Chern JJ, Relyea K, Jea A. Complications of occipital screw placement for occipitocervical fusion in children. J Neurosurg Pediatr 2012; 9:586-593.

11. Nockels RP, Shaffrey CI, Kanter AS, Azeem S, York JE. Occipitocervical fusion with rigid internal fixation: long-term follow-up data in 69 patients. J Neurosurg Spine 2007; 7:117123. 